\title{
On Contact Processes in Continuum
}

\section{Yuri Kondratiev}

Department of Mathematics and BiBoS, Bielefeld University

Postfach 1001 31, D-33501 Bielefeld, Germany; National University "Kiev Mohyla Academy", Ukraine

e-mail: kondrat@mathematik.uni-bielefeld.de

\section{Anatoli Skorokhod}

Department of Statistics and Probability, Michigan State University, E.Lansing, MI 48824, USA; Institute of Mathematics of NASU, Kiev, Ukraine

e-mail: skorokhod@stt.msu.edu

\begin{abstract}
We introduce a continuous version of the contact model which is well-known and widely studied in the lattice case. Under certain general assumptions on the infection spreading characteristics we construct the contact process as a Markov process in the configuration space of the system.
\end{abstract}

2000 AMS Mathematics Subject Classification: 60K35, 60J75, 60J80, 82C21, 82C22

Keywords: Birth-and-death process; Continuous system; Contact model

\section{Introduction}

Let us start with a reminder of some facts from the theory of stochastic lattice gases on the cubic lattice $\mathbb{Z}^{d}, d \in \mathbb{N}$. In the lattice gas model with spin space $S=\{0,1\}$, the configuration space is defined as $\mathcal{X}=\{0,1\}^{\mathbb{Z}^{d}}$. For a given $\sigma=\left\{\sigma(x) \mid x \in \mathbb{Z}^{d}\right\} \in \mathcal{X}$ we say that a lattice side $y \in \mathbb{Z}^{d}$ is free or occupied by a particle depending on $\sigma(y)=0$ or $\sigma(y)=1$ respectively.

In the Glauber type stochastic dynamics of the lattice gas particles randomly disappear from occupied sites or appear of free places of the lattice. Obviously, this dynamics may be interpreted as a birth-and-death process on $\mathbb{Z}^{d}$. The generator of this dynamics is given by

$$
(L f)(\sigma)=\sum_{x \in \mathbb{Z}^{d}} a(x, \sigma)\left(\nabla_{x} f\right)(\sigma)
$$


where

$$
\left(\nabla_{x} f\right)(\sigma)=f\left(\sigma^{x}\right)-f(\sigma)
$$

$\sigma^{x}$ denoting the configuration $\sigma$ in which the particle at site $x$ has changed its spin value.

An extremely important example of such type stochastic dynamics is given by the classical contact model. In the model $\sigma \rightarrow \sigma^{x}$ at rate 1 if $\sigma(x)=1$ and at rate $\lambda|\{y|| y-x \mid=1\}|$ if $\sigma(x)=0$, where $|A|$ denotes the cardinality of the set $A$. The interpretation is that sites with $\sigma(x)=1$ are infected while sites with $\eta(x)=0$ are healthy. Infected sites recover from the infection after an exponential time of rate 1 , while healthy sites became be infected at a rate proportional to the number of infected neighbors. The contact model is one of the simplest ones in the theory of interacting particle systems. But even this simple model has very reach and interesting properties, especially, concerning the asymptotic behavior and the structure of equilibrium measures, see [6], [7].

If we consider a continuous particle system, i.e., a system of particles which can take any position in the Euclidean space $\mathbb{R}^{d}$, then an analog of the discussed lattice stochastic dynamics should be a process in which particles randomly appear and disappear in the space, i.e., a spatial birth-and-death process. The generator of such a process is informally given by the formula

$$
(L F)(\gamma)=\sum_{x \in \gamma} d(x, \gamma)\left(D_{x}^{-} F\right)(\gamma)+\int_{\mathbb{R}^{d}} b(x, \gamma)\left(D_{x}^{+} F\right)(\gamma) d x
$$

where

$$
\left(D_{x}^{-} F\right)(\gamma)=F(\gamma \backslash x)-F(\gamma), \quad\left(D_{x}^{+} F\right)(\gamma)=F(\gamma \cup x)-F(\gamma)
$$

Here and below, for simplicity of notations, we just write $x$ instead of $\{x\}$. The coefficient $d(x, \gamma)$ describes the rate at which the particle $x$ of the configuration $\gamma$ dies, while $b(x, \gamma)$ describes the rate at which, given the configuration $\gamma$, a new particle is born at $x$.

Spatial birth-and-death processes were first discussed by Preston in [8]. Under some conditions on the birth and death rates, Preston proved the existence of such processes in a bounded domain in $\mathbb{R}^{d}$. Though the number of particles can be arbitrarily large in this case, the total number of particles remains finite at any moment of time. The problem of construction of a spatial birth-and-death process in the infinite volume was initiated by Holley and Stroock in [2]. In fact, in that paper, birth-and-death processes in bounded domains were analyzed in detail. Only in a very special case of nearest neighbor birth-and-death processes on the real line, the existence of a corresponding process on the whole space was proved and its properties were studied. For the particular case of the Glauber stochastic dynamics in continuum (in which we have an a priori given stationary measure) so-called equilibrium Markov processes in infinite volume were constructed in [4]. A general case of birth-and-death processes in unbounded domains is considered in our forthcoming paper [5]. 
In this paper we introduce a continuous version of the contact model described above. This model can be interpreted also as a spatial infinite particle branching model with killing. Our first result (Theorem 3.1) shows that for a bounded contact rate and for a large set of initial configurations there exists corresponding contact process as a Markov process in the configuration space of the system. Conditions on the infection spreading rate are quite general. Only one essential assumption should be mentioned: we assume a finite range of the influence of infected points in the infection spreading process. The second result is related with the case of possible unbounded contact rate but still under the compact support assumption (Theorem 3.2). There we should modify the phase space of the contact process depending on the integrability property of the contact rate s.t. corresponding process will not leave this (bigger) phase space under the stochastic evolution.

\section{Configuration spaces}

The configuration space $\Gamma:=\Gamma\left(\mathbb{R}^{d}\right)$ over $\mathbb{R}^{d}$ is defined as the set of all subsets of $\mathbb{R}^{d}$ which are locally finite:

$$
\Gamma:=\left\{\gamma \subset \mathbb{R}^{d}:\left|\gamma_{\Lambda}\right|<\infty \text { for each compact } \Lambda \subset \mathbb{R}^{d}\right\},
$$

where $|\cdot|$ denotes the cardinality of a set and $\gamma_{\lambda}:=\gamma \cap \Lambda$. One can identify any $\gamma \in \Gamma$ with the positive Radon measure $\sum_{x \in \gamma} \varepsilon_{x} \in \mathcal{M}\left(\mathbb{R}^{d}\right)$, where $\varepsilon_{x}$ is the Dirac measure with mass at $x, \sum_{x \in \varnothing} \varepsilon_{x}:=$ zero measure, and $\mathcal{M}\left(\mathbb{R}^{d}\right)$ stands for the set of all positive Radon measures on $\mathcal{B}\left(\mathbb{R}^{d}\right)$. The space $\Gamma$ can be endowed with the relative topology as a subset of the space $\mathcal{M}\left(\mathbb{R}^{d}\right)$ with the vague topology, i.e., the weakest topology on $\Gamma$ with respect to which all maps

$$
\Gamma \ni \gamma \mapsto\langle f, \gamma\rangle:=\int_{\mathbb{R}^{d}} f(x) \gamma(d x)=\sum_{x \in \gamma} f(x), \quad f \in C_{0}\left(\mathbb{R}^{d}\right),
$$

are continuous. Here, $C_{0}\left(\mathbb{R}^{d}\right)$ is the space of all continuous real-valued functions on $\mathbb{R}^{d}$ with compact support. We shall denote the Borel $\sigma$-algebra on $\Gamma$ by $\mathcal{B}(\Gamma)$. In the sequel, we will use equally both interpretations for configurations that does not produce any confusion because it will be always clear from the context.

The vague topology on $\Gamma$ is metrizable with the metric

$$
d_{w}\left(\gamma, \gamma^{\prime}\right)=\sum_{n}\left(d_{w}^{(n)}\left(\gamma_{B_{n}(0)}, \gamma_{B_{n}(0)}^{\prime}\right) \wedge 1\right) 2^{-n}
$$

where $d_{w}^{(n)}$ is the metric in the space $\mathcal{M}\left(B_{n}(0)\right)$ of the finite measures on the ball $B_{n}(0)$ in $\mathbb{R}^{d}$ of the radius $n \in \mathbb{N}$ with the center at point $0 \in \mathbb{R}^{d}$. In this metric $\Gamma$ is not 
complete. But it is possible to modify this metric in such a way that the space $\Gamma$ with the new metric $d_{\Gamma}$ became be a complete separable metric space, see, e.g., [3].

Let us describe compacts in the configuration space. Introduce a sequences of functions on $\Gamma$ :

$$
\delta_{n}(\gamma)=\inf \left\{\left|x-x^{\prime}\right| \mid x, x^{\prime} \in \gamma_{B_{n}(0)}, x \neq x^{\prime}\right\}, n \in \mathbb{N} .
$$

Lemma 2.1 A set $F \subset \Gamma$ is a relatively compact set iff for any $n \in \mathbb{N}$

$$
\sup _{\gamma \in F}\left\{\left|\gamma_{B_{n}(0)}\right|+\delta_{n}^{-1}(\gamma)\right\}<\infty
$$

For the proof see [2], [3].

We denote by $\mathcal{K}(\Gamma)$ the set of all relatively compact subsets of $\Gamma$.

For any $\beta>0$ introduce a function $e_{\beta}(x):=e^{-\beta|x|}, x \in \mathbb{R}^{d}$ and symmetric kernel

$$
\Psi_{\beta}(x, y):=e^{-\beta|x|} e^{-\beta|y|} \frac{|x-y|+1}{|x-y|} \mathbf{1}_{\{x \neq y\}}
$$

on $\mathbb{R}^{d} \times \mathbb{R}^{d}$. We construct the following functions on $\Gamma$ :

$$
\begin{gathered}
\mathbb{L}_{\beta}(\gamma):=<e_{\beta}(x), \gamma>=\sum_{x \in \gamma} e^{-\beta|x|}(\leq+\infty), \gamma \in \Gamma, \\
\mathbb{E}_{\beta}(\gamma):=\frac{1}{2} \iint \Psi_{\beta}(x, y) \gamma(d x) \gamma(d y)=\sum_{\{x, y\} \subset \gamma} \Psi_{\beta}(x, y)(\leq+\infty), \gamma \in \Gamma, \\
\mathbb{V}_{\beta}(\gamma):=\mathbb{L}_{\beta}(\gamma)+\mathbb{E}_{\beta}(\gamma), \gamma \in \Gamma,
\end{gathered}
$$

Following lemma is a particular case of a general statement about compact functions on $\Gamma$, for the proof see [3],[5].

Lemma 2.2 For any $C \in \mathbb{R}_{+}$holds

$$
\left\{\gamma \in \Gamma \mid \mathbb{V}_{\beta} \leq C\right\} \in \mathcal{K}(\Gamma) .
$$

Denote

$$
\Gamma_{\beta}:=\left\{\gamma \in \Gamma \mid \mathbb{V}_{\beta}(\gamma)<+\infty\right\} .
$$

Then for $\beta<\beta^{\prime}$ we have $\Gamma_{\beta} \subset \Gamma_{\beta^{\prime}}$. Due to Lemma $2.2 \Gamma_{\beta}$ is a $\sigma$-compact set. Introduce subspace

$$
\Gamma_{\infty}:=\bigcup_{\beta>0} \Gamma_{\beta} \subset \Gamma
$$


Remark 2.1 The subspace $\Gamma_{\infty}$ is massive enough to contain supports for a large class of probability measures on $\mathcal{B}(\Gamma)$. Namely, for a probability measure $\mu \in \mathcal{M}^{1}(\Gamma)$ we assume the existence of second local moments, that is the condition

$$
\forall \Lambda \subset \mathbb{R}^{d}, \text { compact } \int_{\Lambda}\left|\gamma_{\Lambda}\right|^{2} \mu(d \gamma)<\infty
$$

Define first and second correlation measures on $\mathcal{B}\left(\mathbb{R}^{d}\right)$ and $\mathcal{B}\left(\mathbb{R}^{d} \times \mathbb{R}^{d}\right)$ respectively via the following relations

$$
\begin{gathered}
\int_{\Gamma} \sum_{x \in \gamma} f(x) \mu(d \gamma)=\int_{\mathbb{R}^{d}} f(x) \rho^{(1)}(d x), \\
\int_{\Gamma} \sum_{\{x, y\} \subset \gamma} g(x, y) \mu(d \gamma)=\int_{\mathbb{R}^{d}} \int_{\mathbb{R}^{d}} g(x, y) \rho^{(2)}(d x, d y),
\end{gathered}
$$

for any function $f \in C_{0}\left(\mathbb{R}^{d}\right)$ and any symmetric kernel $g \in C_{0}\left(\mathbb{R}^{d} \times \mathbb{R}^{d}\right)$. Then

$$
\int_{\Gamma} \mathbb{V}_{\beta}(\gamma) \mu(d \gamma)=\int_{\mathbb{R}^{d}} e_{\beta}(x) \rho^{(1)}(d x)+\int_{\mathbb{R}^{d}} \int_{\mathbb{R}^{d}} \Psi_{\beta}(x, y) \rho^{(2)}(d x, d y)
$$

and the finiteness of the last expression will imply $\mu\left(\Gamma_{\beta}\right)=1$. In particular, assume that the first and second correlation measure are absolutely continuous w.r.t. the Lebesgue measure, i.e.,

$$
\rho^{(1)}(d x)=k^{(1)}(x) d x
$$

$\left(k^{(1)}(x)\right.$ is called first correlation function) and

$$
\rho^{(2)}(d x, d y)=\frac{1}{2} k^{(2)}(x, y) d x d y
$$

with a symmetric function $k^{(2)}(x, y)$ (so-called second correlation function of the measure $\mu)$. Then sub-exponential bound

$$
k^{(1)}(x) \leq A \exp (B|x|), k^{(2)}(x, y) \leq A \exp (B|x|+B|y|), x, y \in \mathbb{R}^{d},
$$

with some $A, B>0$ imply

$$
\int_{\mathbb{R}^{d}} e_{\beta}(x) k^{(1)}(x) d x+\int_{\mathbb{R}^{d}} \int_{\mathbb{R}^{d}} \Psi_{\beta}(x, y) k^{(2)}(x, y) d x d y<\infty
$$

for all $\beta>B$ taking into account

$$
\int_{B_{1}(0)} \int_{B_{1}(0)} \frac{d x d y}{|x-y|}<\infty
$$

for any $d \in \mathbb{N}$. Therefore, $\mu\left(\Gamma_{\infty}\right)=1$ for any such measure $\mu$. 


\section{Contact models}

Contact models we are going to discuss can be considered as a particular case of general birth-and-death Markov processes in configuration spaces. These spatial birth-anddeath processes describe a stochastic evolutions in $\Gamma$ in which points of a configuration can randomly appear and disappear. The generator of such process is heuristically given on functions $F: \Gamma \rightarrow \mathbb{R}$ by

$$
\left(L_{\mathrm{b}, \mathrm{d}} F\right)(\gamma)=\sum_{x \in \gamma} d(x, \gamma \backslash x)\left(D_{x}^{-} F\right)(\gamma)+\int_{\mathbb{R}^{d}} b(x, \gamma)\left(D_{x}^{+} F\right)(\gamma) d x
$$

where

$$
\left(D_{x}^{-} F\right)(\gamma)=F(\gamma \backslash x)-F(\gamma), \quad\left(D_{x}^{+} F\right)(\gamma)=F(\gamma \cup x)-F(\gamma)
$$

Here and below, for simplicity of notations, we just write $x$ instead of $\{x\}$. The coefficient $d(x, \gamma)$ describes the rate at which the particle $x \in \gamma$ of the configuration $\gamma$ dies, while $b(x, \gamma)$ describes the rate at which, given the configuration $\gamma$, a new particle is born at $x \in \mathbb{R}^{d}$.

To give a meaning to such type operators, let us introduce the set $\mathcal{F} C_{\mathrm{b}}\left(C_{0}\left(\mathbb{R}^{d}\right), \Gamma\right)$ of all continuous bounded cylinder (or finitely based) functions on $\Gamma$. These functions have the following form

$$
\Gamma \ni \gamma \mapsto F(\gamma)=g_{F}\left(\left\langle\varphi_{1}, \gamma\right\rangle, \ldots,\left\langle\varphi_{N}, \gamma\right\rangle\right)
$$

where $N \in \mathbb{N}, \varphi_{1}, \ldots, \varphi_{N} \in C_{0}\left(\mathbb{R}^{d}\right)$ and $g_{F} \in C_{\mathrm{b}}\left(\mathbb{R}^{N}\right)$, where $C_{\mathrm{b}}\left(\mathbb{R}^{N}\right)$ denotes the set of all continuous bounded functions on $\mathbb{R}^{N}$. Then under a local integrability (in $x \in \mathbb{R}^{d}$ ) condition on the birth rate the expression (3.1) makes sense al least pointwisely on $\Gamma$.

In the study of an infection spreading inside of a population is naturally to consider a continuous version of the (well-known in the lattice case) contact model. This model can be described as a birth-and-death process with the death rate $d \equiv 1$ and the birth rate

$$
\begin{gathered}
b(x, \gamma)=\lambda \sum_{y \in \gamma} a(x-y), \\
0 \leq a \in L^{1}\left(\mathbb{R}^{d}\right),\|a\|_{1}=1,
\end{gathered}
$$

where $\lambda>0$ denotes the infection spreading rate. In the contact model a configuration describes the set of infected individuals in a population. These individuals became be healthy after a random exponentially distributed time independently on others (that correspond to disappearing of a point from the configuration) or there can appear a new infected individual at a point $x$ with the rate (3.3) depending on the presence of infected ones around this point.

Another interpretation of this process is related to population biology models. In such a process we have some kind of spatial branching with killing: each particle $y \in \gamma$ 
creates (independently of others) a new particle at point $x \in \mathbb{R}^{d}$ with the rate $\lambda a(x-y) d x$ and each particle dies independently of others after a random exponentially distributed time. This interpretation is motivated by the form of generator of the contact process:

$$
L F(\gamma)=\sum_{x \in \gamma}(F(\gamma \backslash x)-F(\gamma))+\lambda \sum_{y \in \gamma} \int_{\mathbb{R}^{d}} a(x-y)[F(\gamma \cup x)-F(\gamma)] d x
$$

in which the additive character of the birth rate is essential. We will call the birth coefficient (3.3) in the contact model the linear birth rate. In our forthcoming paper [5] we discuss general birth-and-death processes with sub-linear rates.

Theorem 3.1 Let $d \geq 2$ and $a \in L^{\infty}\left(\mathbb{R}^{d}\right)$ has bounded support. Then for any initial configuration $\gamma \in \Gamma_{\infty}$ there exists a Markov function $X_{t}^{\gamma}, t \geq 0$, with the transition probability corresponding to generator (3.5) which starts with $\gamma$ and such that

$$
\forall t \geq 0 X_{t}^{\gamma} \in \Gamma_{\infty} \text { a.s. }
$$

Proof. Denote $\Gamma_{0} \subset \Gamma$ the set of all finite configurations in $\mathbb{R}^{d}$. This set has a decomposition into disjunct union

$$
\Gamma_{0}=\bigsqcup_{n=0}^{\infty} \Gamma^{(n)}, \quad \Gamma^{(n)}=\{\gamma \in \Gamma|| \gamma \mid=n\}, n \in \mathbb{N}, \Gamma^{(0)}=\{\varnothing\} .
$$

The topology of $\Gamma_{0}$ is naturally coming from the topology of disjunct components.

We would like to show first that for any starting point $\gamma \in \Gamma_{0}$ the contact process exists as a Markov process in $\Gamma_{0}$. To this end we rewrite the generator (3.5) in the standard form of a pure jump Markov generator. Namely, using explicit form of $L$ we have for any finite configuration $\eta \in \Gamma_{0}$

$$
L F(\eta)=\lambda(\eta)\left\{\int_{\Gamma_{0}} F\left(\eta^{\prime}\right) Q\left(\eta, d \eta^{\prime}\right)-F(\eta)\right\},
$$

where $\lambda(\eta)=|\eta|(1+\lambda)$ and the transition kernel $Q\left(\eta, d \eta^{\prime}\right)$ on $\Gamma_{0}$ is given by

$$
Q\left(\eta, d \eta^{\prime}\right)=\frac{1}{\lambda(\eta)}\left\{\sum_{x \in \eta} \delta_{\eta \backslash x}\left(d \eta^{\prime}\right)+\lambda \sum_{y \in \eta} \int_{\mathbb{R}^{d}} a(x-y) \delta_{\eta \cup x}\left(d \eta^{\prime}\right) d x\right\} .
$$

An application of the pure jump Markov processes theory gives us the existence of of a probability space $(\Omega, \mathcal{F}, P)$ and the Markov process $X_{t}^{\gamma}(\omega), t<\zeta_{\infty}(\omega)$, in the state space $\Gamma_{0}$ with the generator $L$, where $\zeta_{\infty}(\omega)$ is the life time of the process, see, e.g., [1]. Let us show regularity of this process, i.e., the relation

$$
P\left(\left\{\omega \in \Omega \mid \zeta_{\infty}(\omega)=+\infty\right\}\right)=1 .
$$


Note that for the function

$$
\Gamma_{0} \ni \eta \mapsto|\eta| \in \mathbb{R}_{+}
$$

holds

$$
L|\eta|=-|\eta|+\lambda \sum_{y \in \eta} \int_{\mathbb{R}^{d}} a(x-y) d x=(\lambda-1)|\eta| \leq \lambda|\eta| .
$$

Then the representation

$$
\left|X_{t}^{\gamma}\right|=|\gamma|+\int_{0}^{t} L\left|X_{s}^{\gamma}\right| d s+M_{t}
$$

where $M_{t}$ is a martingale, together with the Gronwall inequality imply

$$
\mathbb{E}\left|X_{t}^{\gamma}\right| \leq|\gamma| e^{\lambda t}
$$

The latter shows the regularity of the process.

Now we are ready to construct the infinite particle contact process.

For any given initial configuration $\gamma \in \Gamma_{\infty}$ there exists $\beta>0$ such that $\gamma \in \Gamma_{\beta}$. Denote $\gamma_{n}:=\gamma \cap B_{n}(0), n \in \mathbb{N}$ and $X_{t}^{n}:=X_{t}^{\gamma_{n}}$. The sequence of Markov processes $\left\{X_{t}^{n} \mid n \in \mathbb{N}\right\}$ in $\Gamma_{0}$ with the same generator $L$ has following monotonicity property:

$$
\forall n \in \mathbb{N} \quad \forall t \geq 0 \quad X_{t}^{n} \subset X_{t}^{n+1} \quad \text { a.s. }
$$

This property is a consequence of the observation that for a decomposition of initial configuration in $B_{n+1}(0)$

$$
\gamma_{n+1}=\gamma_{n} \cup \eta_{n}, \quad \eta_{n}=\gamma_{n+1} \backslash \gamma_{n}
$$

we have

$$
X_{t}^{n+1}=X_{t}^{n} \cup X_{t}^{\eta_{n}} \quad \text { a.s. }
$$

Really, due to the additive structure of the generator 3.5, $X_{t}^{n}$ and $X_{t}^{\eta_{n}}$ are independent Markov processes of spatial branching with killing and $X_{t}^{n} \cap X_{t}^{\eta_{n}}=\varnothing$ a.s.

Introduce limiting process

$$
X_{t}^{\gamma}(\omega)=\bigcup_{n=1}^{\infty} X_{t}^{n}(\omega)
$$

The following lemma shows that the function $\mathbb{V}_{\beta}$ can be considered as a Lyapunov function for the generator $L$.

Lemma 3.1 There exists a constant $C>0$ such that

$$
L \mathbb{V}_{\beta}(\gamma) \leq C \mathbb{V}_{\beta}(\gamma), \quad \gamma \in \Gamma_{\beta}
$$


Proof. Let us take any $\gamma \in \Gamma_{0}$. Using 3.5 we obtain

$$
\begin{gathered}
L \mathbb{L}_{\beta}(\gamma)=-\mathbb{L}_{\beta}(\gamma)+\lambda \sum_{y \in \gamma} \int_{\mathbb{R}^{d}} a(x) \exp (-\beta|x-y|) d x \leq \\
-\mathbb{L}_{\beta}(\gamma)+\lambda \sum_{y \in \gamma} \int_{B_{R}(0)} a(x) \exp (-\beta|x-y|) d x \leq \\
-\mathbb{L}_{\beta}(\gamma)+C_{1} \sum_{y \in \gamma} \exp (-\beta|y|)=C_{2} \mathbb{L}_{\beta}(\gamma),
\end{gathered}
$$

where we have used a compact support property of $a$.

Similarly,

$$
L \mathbb{E}_{\beta}(\gamma)=-\sum_{x \in \gamma} \sum_{y \in \gamma \backslash x} \Psi_{\beta}(x, y)+\lambda \sum_{y \in \gamma} \sum_{z \in \gamma} \int_{\mathbb{R}^{d}} a(x-y) \Psi_{\beta}(x, z) d x .
$$

Using again compact support property and boundedness of $a$ and explicit form of $\Psi_{\beta}$ we can estimate the latter by

$$
C_{3} \sum_{y \in \gamma} \sum_{z \in \gamma} \exp (-\beta|y|) \exp (-\beta|z|) \int_{B_{R}(0)} \frac{1}{|x-y-z|} d x .
$$

The integral over $B_{R}(0)$ in this expression is uniformly bonded in $y, z \in \mathbb{R}^{d}$ for $d \geq 2$ that gives

$$
\begin{gathered}
L \mathbb{E}_{\beta}(\gamma) \leq C_{4} \sum_{y \in \gamma} \sum_{z \in \gamma} \exp (-\beta|y|) \exp (-\beta|z|)= \\
2 C_{4} \sum_{\{x, y\} \subset \gamma} \exp (-\beta|y|) \exp (-\beta|z|)+C_{4} \sum_{y \in \gamma} \exp (-2 \beta|y|) \leq \\
2 C_{4} \mathbb{E}_{\beta}(\gamma)+C_{4} \mathbb{L}_{\beta}(\gamma) \leq C_{5} \mathbb{V}_{\beta}(\gamma) .
\end{gathered}
$$

Obtained estimations prove the statement of the lemma for $\gamma \in \Gamma_{0}$. The general case follows for any $\gamma \in \Gamma_{\beta}$ by the consideration of the monotonically growing approximate sequence $\left\{\gamma_{n}=\gamma \cap B_{n}(0) \mid n \in \mathbb{N}\right\} \subset \Gamma_{0}$ with a use of monotonicity arguments in the expressions for $\mathbb{V}_{\beta}\left(\gamma_{n}\right)$ and $L \mathbb{V}_{\beta}\left(\gamma_{n}\right)$ (see calculation above)

Consider now $\mathbb{V}_{\beta}\left(X_{t}^{n}(\omega)\right)$. Due to the Markov property we have

$$
\begin{gathered}
\mathbb{E}\left[\mathbb{V}_{\beta}\left(X_{t}^{n}\right)\right]=\mathbb{V}_{\beta}\left(\gamma_{n}\right)+\int_{0}^{t} \mathbb{E}\left[L \mathbb{V}_{\beta}\left(X_{s}^{n}\right)\right] d s \leq \\
\mathbb{V}_{\beta}(\gamma)+C \int_{0}^{t} \mathbb{E}\left[\mathbb{V}_{\beta}\left(X_{s}^{n}\right)\right] d s
\end{gathered}
$$


where we have used Lemma 3.1. The Gronwall inequality gives then

$$
\forall n \in \mathbb{N} \forall t \geq 0 \quad \mathbb{E}\left[\mathbb{V}_{\beta}\left(X_{t}^{n}\right)\right] \leq \mathbb{V}_{\beta}(\gamma) e^{C t} .
$$

The bound (3.10) gives due to a.s. monotonicity in $n$ of the sequence of processes $X_{t}^{n}$

$$
\mathbb{E}\left[\mathbb{V}_{\beta}\left(X_{t}\right)\right] \leq \mathbb{V}_{\beta}(\gamma) e^{C t}
$$

that means $\forall t \geq 0 X_{t} \in \Gamma_{\beta}$ a.s. Due to the construction, the Markov process $X_{t}^{\gamma}$ has the generator $L$ and $X_{0}^{\gamma}=\gamma$.

Remark 3.1 From the proof of the theorem follows that the restriction $d \geq 2$ is related only with the needing integrability

$$
\int_{B_{R}(0)} \frac{d x}{|x|}<\infty
$$

For the case $d=1$ we can just modify the definition of the kernel $\Psi_{\beta}$ taking in the definition $|x-y|^{\delta}$ with some $\delta<1$ instead $|x-y|$. Then the presented proof works also for the one-dimensional contact model.

Remark 3.2 Let $\mu_{0} \in \mathcal{M}^{1}(\gamma)$ be an initial distribution on $\mathcal{B}(\Gamma)$ s.t. $\mu_{0}\left(\Gamma_{\infty}\right)=1$ and $X_{t} \in \Gamma$ denotes the contact Markov process with this initial measure. Let $k_{t}(x), x \in \mathbb{R}^{d}$, denotes the density of the infected population at the time $t$ (i.e., the first correlation function of the distribution $\mu_{t}$ on $\Gamma$ of the process $X_{t}$ ). Then the definition of the process shows that this density satisfies the following Cauchy problem:

$$
\frac{\partial k_{t}(x)}{\partial t}=-k_{t}(x)+\lambda\left(k_{t} * a\right)(x)
$$

with the initial value $k_{0}(x)$ that is the first correlation function for $\mu_{0}$ (density of the initial infected population) which we can assume be bounded and continuous.

This equation can be rewritten as

$$
\frac{\partial k_{t}(x)}{\partial t}=\left(\lambda L_{a}-C\right) k_{t}(x)
$$

where

$$
\left(L_{a} f\right)(x)=\int_{\mathbb{R}^{d}} a(x-y)[f(y)-f(x)] d y,
$$

is the generator of a pure jump Markov process in $\mathbb{R}^{d}$ and $C=1-\lambda$. Due to this equation we see 3 possible cases:

(i) $\lambda<1$ (subcritical). In this case $k_{t} \rightarrow 0, t \rightarrow \infty$ uniformly on $\mathbb{R}^{d}$. Therefore, the infected population will be asymptotically degenerated.

(ii) $\lambda>1$ (supercritical). Here $k_{t} \rightarrow+\infty, t \rightarrow \infty$ pointwisely exponentially fast. 
(iii) $\lambda=1$ (critical). Now the time evolution of the density is describing by the Kolmogorov equation for the mentioned jump process:

$$
\frac{\partial k_{t}(x)}{\partial t}=L_{a} k_{t}(x)
$$

and its asymptotic is nontrivial. From the point of view of applications, the asymptotic of the distribution $\mu_{t}$ and the problem of the existence of corresponding invariant distribution are most interesting open questions. These problems we study in a separated work.

Theorem 3.1 gives the existence of the contact process in the case of a bounded contact rate $a$ which has compact support. Let us consider more general case of a (possible unbounded) rate $a$.

To this end we will need to introduce some additional topological structures on $\Gamma$.

For any $\beta>0$ and $\alpha>0$ we introduce the exponential function $e_{\beta}(x):=e^{-\beta|x|}, x \in$ $\mathbb{R}^{d}$ and the kernel

$$
\Psi_{\beta}^{\alpha}(x, y):=e^{-\beta|x|} e^{-\beta|y|} \frac{|x-y|^{\alpha}+1}{|x-y|^{\alpha}} \mathbf{1}_{\{x \neq y\}}, x, y \in \mathbb{R}^{d} .
$$

Then we construct following functions on $\Gamma$ :

$$
\begin{gathered}
\mathbb{L}_{\beta}(\gamma):=<e_{\beta}(x), \gamma>= \\
\sum_{x \in \gamma} e^{-\beta|x|}(\leq+\infty), \gamma \in \Gamma, \\
\mathbb{E}_{\beta}^{\alpha}(\gamma):=\frac{1}{2} \iint \Psi_{\beta}^{\alpha}(x, y) \gamma(d x) \gamma(d y)= \\
\sum_{\{x, y\} \subset \gamma} \Psi_{\beta}^{\alpha}(x, y)(\leq+\infty), \gamma \in \Gamma,
\end{gathered}
$$

and introduce a generalization of function (2.5) as follows:

$$
\mathbb{V}_{\beta}^{\alpha}(\gamma):=\mathbb{L}_{\beta}(\gamma)+\mathbb{E}_{\beta}^{\alpha}(\gamma), \gamma \in \Gamma
$$

Similarly to Lemma 2.2 we can show that it is a compact functions on $\Gamma$. Namely, for any $C>0$ holds

$$
\left\{\gamma \in \Gamma \mid \mathbb{V}_{\beta}^{\alpha} \leq C\right\} \in \mathcal{K}(\Gamma)
$$

Denote

$$
\Gamma_{\beta}^{\alpha}:=\left\{\gamma \in \Gamma \mid \mathbb{V}_{\beta}^{\alpha}(\gamma)<+\infty\right\}
$$

It is easy to see that 


$$
\beta<\beta^{\prime} \Longrightarrow \Gamma_{\beta}^{\alpha} \subset \Gamma_{\beta^{\prime}}^{\alpha}
$$

and

$$
\alpha<\alpha^{\prime} \Longrightarrow \Gamma_{\beta}^{\alpha^{\prime}} \subset \Gamma_{\beta}^{\alpha}
$$

For given $\alpha, \beta>0$ the subspace $\Gamma_{\beta}^{\alpha}$ is a $\sigma$-compact set. Denote

$$
\Gamma_{\infty}^{0}:=\bigcup_{\alpha>0} \bigcup_{\beta>0} \Gamma_{\beta}^{\alpha} \subset \Gamma
$$

Theorem 3.2 Let

$$
a \in L^{1+\delta}\left(\mathbb{R}^{d}\right)
$$

for some $\delta>0$ and has bounded support. Then for all

$$
\alpha<\frac{\delta d}{1+\delta}
$$

$\beta>0$ and $\gamma \in \Gamma_{\beta}^{\alpha}$ there exists a Markov function $X_{t}^{\gamma}, t \geq 0$, with the transition probability corresponding to generator (3.5) which starts with $\gamma$ and such that

$$
\forall t \geq 0 \quad X_{t}^{\gamma} \in \Gamma_{\beta}^{\alpha} \text { a.s. }
$$

Proof. The proof of this theorem is similar to the proof of Theorem 3.1 with only one modification. We will use the function $\mathbb{V}_{\beta}^{\alpha}$ as a Lyapunov functional for the generator $L$. It will need an inequality

$$
L \mathbb{V}_{\beta}^{\alpha} \leq C \mathbb{V}_{\beta}^{\alpha}
$$

The latter can be stated as in Lemma 3.1 if we will exploit in the bound for the integral

$$
\int_{\mathbb{R}^{d}} a(x-y) \Psi_{\beta}^{\alpha}(x, z) d x
$$

the compact support property of $a$ and the Hölder inequality instead of the uniform bound on $a$ as before.

Acknowledgments Financial support of the DFG through SFB-701 (Bielefeld University) and the Research Projects 436 UKR 113/70 is gratefully acknowledged. A.S. is thankful to the ZIF, Bielefeld University, for the hospitality during his visit in July 2005 in the framework of the Research Semester "Modelling in the Sciences". 


\section{References}

[1] I.I. Gikhman, A.V. Skorokhod, The Theory of Stochastic Processes, v. 2, SprigerVerlag, 1979.

[2] R. A. Holley, D. W. Stroock, Nearest neighbor birth and death processes on the real line, Acta Math. 140 (1978) 103-154.

[3] Yu. Kondratiev, O. Kutovyi, On topological properties of configuration spaces, BiBoS Preprint, to appear in Math. Nachrichten, 2003.

[4] Yu.Kondratiev, E.Lytvynov, Glauber dynamics of continuous particle systems Ann. Inst. H.Poincare, Ser. B, 41 (2005), 685-702.

[5] Yu.Kondratiev, A.Skorokhod, Birth-and-death Markov processes in configuration spaces, in preparation, 2005.

[6] T. M. Liggett, Interacting Particle Systems, Springer-Verlag, 1985.

[7] T. M. Liggett, Stochastic Interacting Systems: Contact, Voter and Exclusion Processes, Springer-Verlag, 1999.

[8] C. Preston, Spatial birth-and-death processes, in Proceedings of the 40th Session of the International Statistical Institute (Warsaw, 1975), Vol. 2, Bull. Inst. Internat. Statist., Vol. 46, 1975, pp. 371-391. 